DOE/ER/40762-245

UMD-PP-02-020

\title{
Leading Chiral Contributions to the Spin Structure of the Proton
}

\author{
Jiunn-Wei Chen and Xiangdong Ji \\ Department of Physics, University of Maryland, \\ College Park, MD 20742, USA \\ jwchen@physics.umd.edu, xji@physics.umd.edu
}

\begin{abstract}
The leading chiral contributions to the quark and gluon components of the proton spin are calculated using heavy-baryon chiral perturbation theory. Similar calculations are done for the moments of the generalized parton distributions relevant to the quark and gluon angular momentum densities. These results provide useful insight about the role of pions in the spin structure of the nucleon, and can serve as a guidance for extrapolating lattice QCD calculations at large quark masses to the chiral limit.
\end{abstract}


The spin structure of the proton has been an active subject of investigation in nuclear and high energy physics since the EMC (European Muon Collaboration) measurement of the $g_{1}^{p}\left(x, Q^{2}\right)$ structure function in polarized muon deep-inelastic scattering [1]. EMC found that a surprisingly small fraction of the proton spin resides in quark spin - in flat contradiction with the well-known $\mathrm{SU}(6)$ quark model prediction [2]. In the last decade, many followup experiments have been performed at the CERN, SLAC, and DESY laboratories, and the next-to-leading order QCD analyses of these data confirm the original discovery of EMC [3]. The outstanding question is then what are the other contributions to the proton spin? According to quantum chromodynamics (QCD), the angular momentum of a stronginteracting system can be written as a sum of quark spin $\left(\mathbf{S}_{q}\right)$, quark orbital $\left(\mathbf{L}_{q}\right)$, and gluon angular momentum $\left(\mathbf{J}_{g}\right)$ contributions 喵,

$$
\begin{aligned}
\mathbf{J}_{\mathrm{QCD}}= & \int d^{3} x \frac{1}{2} \psi^{\dagger} \mathbf{\Sigma} \psi+\int d^{3} x \psi^{\dagger} \mathbf{x} \times(-i \mathbf{D}) \psi \\
& +\int d^{3} x \mathbf{x} \times(\mathbf{E} \times \mathbf{B}),
\end{aligned}
$$

where $\mathbf{D}=\partial+i g \mathbf{A}$ is the covariant derivative, $\mathbf{A}$ the gluon potentials, $\psi$ the quark fields, and $\mathbf{E}$ and $\mathbf{B}$ the chromoelectric and magnetic fields. Summations over flavors and colors are implicit. Using the above operator, one can write down a gauge-invariant, frame-independent decomposition of the proton spin [4],

$$
\frac{1}{2}=\frac{1}{2} \Delta \Sigma\left(\mu^{2}\right)+L_{q}\left(\mu^{2}\right)+J_{g}\left(\mu^{2}\right),
$$

in a class of frames in which the proton has a definite helicity [5]. Individual terms in the above equation are defined as the expectation values of the corresponding operators in the proton helicity eigenstate. Because these operators are not separately conserved, their matrix elements depend on renormalization schemes and the related scale $\mu^{2}$.

Recent analyses of the polarized deep-inelastic scattering data yield [3],

$$
\Delta \Sigma\left(\mu^{2} \sim 5 \mathrm{GeV}^{2}\right)=0.16 \pm 0.08 .
$$

Meanwhile, it has been realized that the total quark and gluon contributions to the proton spin, $J_{q}=\Delta \Sigma / 2+L_{q}$ and $J_{g}$, can be obtained from new sum rules involving generalized (off-forward) parton distributions 四. These new distributions are found to be accessible experimentally in deeply virtual Compton scattering [ [0] or exclusive meson production [6]. On the other hand, the matrix elements $\Delta \Sigma, L_{q}$ and $J_{g}$ can be calculated theoretically in lattice QCD and QCD sum rule methods [7]. In the former case, the up and down quark masses used in the present simulations are much larger than the physical ones, and the corresponding pion mass is larger than $500 \mathrm{MeV}$. Therefore, it is crucial to make reliable extrapolations from the lattice data down to the physical pion mass.

In this paper, we study the pionic contribution to the spin structure of the proton. This contribution can be calculated because pions are Goldstone bosons from the spontaneous breaking of the chiral symmetry present in the QCD lagrangian. The explicit breaking of the symmetry through small up and down quark masses induces small physical pion masses. Effective field theory technology allows study of the symmetry breaking effects in a systematic expansion of $m_{\pi} / 4 \pi f_{\pi}$. Here we focus on the leading nonanalytical contributions 
of the type $m_{\pi}^{2} \ln m_{\pi}^{2}$ to the quark and gluon components of the proton spin, $J_{q, g}$. The total nonanalytic contribution to the proton spin, however, must vanish because the total proton spin is independent of the pion mass.

What we actually calculate below is the chiral contribution to the proton form factors of the quark and gluon parts of the QCD energy momentum tensor $T_{q, g}^{\mu \nu}$. A simple counting shows that they have three form factors [4.8],

$$
\begin{aligned}
& \left\langle p^{\prime}\left|T_{q, g}^{(\mu \nu)}\left(\mu^{2}\right)\right| p\right\rangle=\bar{u}\left(p^{\prime}\right)\left[A_{q, g}\left(q^{2}, \mu^{2}\right) \gamma^{(\mu} \bar{p}^{\nu)}\right. \\
& \left.+B_{q, g}\left(q^{2}, \mu^{2}\right) \bar{p}^{(\mu} i \sigma^{\nu) \alpha} q_{\alpha} / 2 M+C_{q, g}\left(q^{2}, \mu^{2}\right) q^{(\mu} q^{\nu)} / M\right] u(p),
\end{aligned}
$$

where $p=\left(p+p^{\prime}\right) / 2, q=p^{\prime}-p$, and $(\cdots)$ means that the indices enclosed are made symmetric and traceless. $A_{q(g)}\left(0, \mu^{2}\right)$ is the momentum fraction of the proton carried by quarks (gluons), and therefore $A_{q}\left(0, \mu^{2}\right)+A_{g}\left(0, \mu^{2}\right)=1$. The quark (gluon) angular momentum contribution to the proton spin is [4]

$$
J_{q, g}\left(\mu^{2}\right)=\frac{1}{2}\left[A_{q, g}\left(0, \mu^{2}\right)+B_{q, g}\left(0, \mu^{2}\right)\right] .
$$

Thus the sum rule $J_{q}\left(\mu^{2}\right)+J_{g}\left(\mu^{2}\right)=1 / 2$ implies $B_{q}\left(0, \mu^{2}\right)+B_{g}\left(0, \mu^{2}\right)=0$. An explicit one-loop verification of this general result in $\mathrm{QED}$ can be found in Ref. [9].

Since we are interested in the form factors $A\left(q^{2}, \mu^{2}\right)$ and $B\left(q^{2}, \mu^{2}\right)$ at zero momentum transfer, chiral perturbation theory is a legitimate tool to use. As a low-energy effective field theory of QCD, chiral perturbation theory treats chiral symmetry and the symmetry breaking patterns of QCD in the most general fashion. In the single nucleon systems, the baryon masses are considered heavy, and the heavy-baryon chiral perturbation theory (HB $\chi \mathrm{PT}$ ) has been formulated to restore the systematic power counting [10]. In this formalism, we rewrite the form factor in the Breit frame as

$$
\begin{aligned}
\left\langle p^{\prime}\left|T_{q, g}^{(\mu \nu)}\left(\mu^{2}\right)\right| p\right\rangle= & \sqrt{1-\frac{q^{2}}{4 M^{2}}} \bar{N}(v)\left\{\left(A_{q, g}\left(q^{2}, \mu^{2}\right)+B_{q, g}\left(q^{2}, \mu^{2}\right) \frac{q^{2}}{4 M^{2}}\right) M v^{(\mu} v^{\nu)}\right. \\
& +\left(A_{q, g}\left(q^{2}, \mu^{2}\right)+B_{q, g}\left(q^{2}, \mu^{2}\right)\right) v^{(\mu}\left[S^{\nu)}, S \cdot q\right] \\
& \left.+C_{q, g}\left(q^{2}, \mu^{2}\right) q^{(\mu} q^{\nu)} / M\right\} N(v),
\end{aligned}
$$

where the proton velocity $v=\left(p+p^{\prime}\right) / 2 M$, polarization vector $S_{\mu}=i \gamma_{5} \sigma_{\mu \nu} v^{\nu}$ reduces to $(0, \vec{\sigma} / 2)$ in the $v^{\mu}=(1,0,0,0)$ frame $(\bar{N} N=2 M)$. In the following, we will focus on the spin-dependent term (the second in the bracket), and compute the chiral corrections to its coefficient, $A_{q, g}(0)+B_{q, g}(0)=2 J_{q, g}$.

According to the formalism developed in Refs. [11], the quark and gluon energymomentum tensor operators are "matched" onto a sum of hadronic operators with the identical quantum numbers, including pure pionic operators, single baryon operators as well as multiple baryon operators,

$$
T_{q, g}^{(\mu \nu)}\left(\mu^{2}\right)=T_{(q, g) \pi}^{(\mu \nu)}\left(\mu^{2}, \Lambda_{\chi}^{2}\right)+T_{(q, g) N}^{(\mu \nu)}\left(\mu^{2}, \Lambda_{\chi}^{2}\right)+T_{(q, g) \Delta}^{(\mu \nu)}\left(\mu^{2}, \Lambda_{\chi}^{2}\right)+\cdots,
$$

where $\Lambda_{\chi}$ is a scale at which $\mathrm{HB} \chi \mathrm{PT}$ is applied. Each of these operators contains an infinite number of terms organized according to the $\mathrm{HB} \chi \mathrm{PT}$ power counting. For instance, the leading terms in the pure pionic operator are 


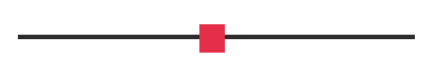

(a)

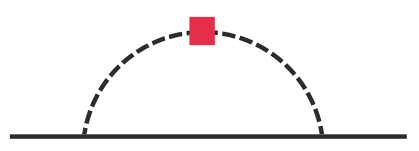

(c)

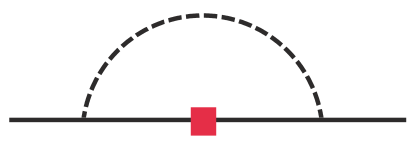

(b)

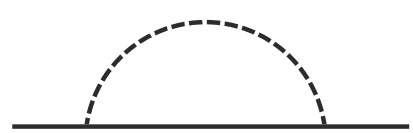

(d)

FIG. 1. Feynman diagrams contributing to the leading chiral behavior of the fractions of the nucleon spin carried in quarks and gluons. The dashed lines represent pions. The diagram (d) denotes the wave function renormalization contribution.

$$
T_{(q, g) \pi}^{(\mu \nu)}\left(\mu^{2}, \Lambda_{\chi}^{2}\right)=a_{(q, g) \pi}\left(\mu^{2}\right) \frac{f_{\pi}^{2}}{2} \operatorname{Tr}\left[\partial^{(\mu} \Sigma^{\dagger} \partial^{\nu)} \Sigma\right]+b_{(q, g) \pi}\left(\mu^{2}\right) \frac{f_{\pi}^{2}}{2} \partial^{(\mu} \operatorname{Tr}\left[\Sigma^{\dagger} \partial^{\nu)} \Sigma+\Sigma \partial^{\nu)} \Sigma^{\dagger}\right]+\cdots
$$

where $\Sigma=u^{2}=\exp \left[i \pi^{a} \tau^{a} / f_{\pi}\right], \pi^{a}$ is the pion field with isospin index $a, \tau^{a}$ the isospin Pauli matrices, $f_{\pi}$ the pion decay constant $=93 \mathrm{MeV}$. The physical meaning of $a_{(q, g) \pi}$ is the momentum fraction of the pion carried by quarks (gluons) in the chiral limit. Hence,

$$
a_{q \pi}\left(\mu^{2}\right)+a_{g \pi}\left(\mu^{2}\right)=1 .
$$

Experimentally, it is found that

$$
a_{q \pi}\left(\mu^{2} \sim 1 \mathrm{GeV}^{2}\right) \equiv\left\langle x_{q}\right\rangle_{\pi} \simeq 0.5
$$

for a physical pion [12]. The leading pionic operator contributes to the leading chiral behavior of the spin fractions $J_{q, g}$ shown in Fig. 1(c).

The leading terms in the single nucleon operator can be written as

$$
\begin{aligned}
T_{(q, g) N}^{(\mu \nu)}\left(\mu^{2}\right)= & a_{(q, g) N}\left(\mu^{2}\right) \bar{N} v^{(\mu} v^{\nu)} N-b_{(q, g) N}\left(\mu^{2}\right) i \partial^{\alpha}\left\{\bar{N} v^{(\mu}\left[S^{\nu)}, S_{\alpha}\right] N\right\} \\
& +c_{(q, g) N}\left(\mu^{2}\right) \bar{N} \mathcal{A}^{(\mu} S^{\nu)} N+\cdots
\end{aligned}
$$

where $\mathcal{A}^{\mu}=i\left(u^{\dagger} \partial^{\mu} u-u \partial^{\mu} u^{\dagger}\right)=-F_{\pi} \tau^{a} \partial^{\mu} \pi^{a}+\cdots$. The second term contributes to $J_{q, g}$ through tree and one loop diagrams (Fig. 1, diagrams (a), (b), and (d)). In fact, $b_{(q, g) N} / 2$ is just $J_{q, g}$ in the limit where quark masses vanish

$$
J_{q, g}^{0}\left(\mu^{2}\right)=b_{(q, g) N}\left(\mu^{2}\right) / 2,
$$




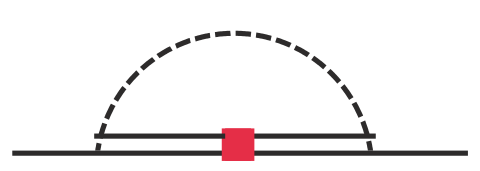

(a)

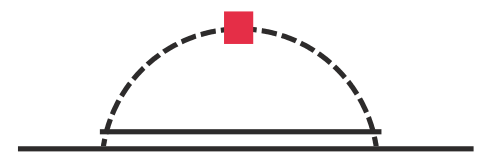

(b)

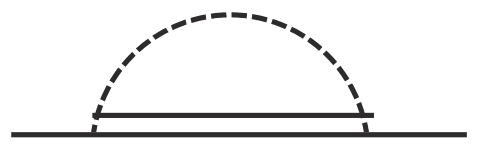

(c)

FIG. 2. Same as Fig. 1. Contributions from the delta intermediate states (double lines). The diagram (c) denotes the wave function renormalization contribution.

where the superscript 0 indicates the chiral limit. The total nucleon momentum and spin in the chiral limit impose the constraints

$$
\begin{gathered}
a_{q N}\left(\mu^{2}\right)+a_{g N}\left(\mu^{2}\right)=1 \\
b_{q N}\left(\mu^{2}\right)+b_{g N}\left(\mu^{2}\right)=1 .
\end{gathered}
$$

Combining the result from different diagrams in Fig. 1, we find the following leading chiral logarithm contributions to the spin structure of the nucleon,

$$
J_{q, g}\left(\mu^{2}\right)=\frac{1}{2}\left\{b_{(q, g) N}\left(\mu^{2}\right)+3\left[a_{(q, g) \pi}\left(\mu^{2}\right)-b_{(q, g) N}\left(\mu^{2}\right)\right] \frac{g_{A}^{2} m_{\pi}^{2}}{\left(4 \pi f_{\pi}\right)^{2}} \ln \left(\frac{m_{\pi}^{2}}{\Lambda_{\chi}^{2}}\right)\right\}+\cdots,
$$

This is the main result of this paper. The chiral logarithms in $J_{q}\left(\mu^{2}\right)+J_{g}\left(\mu^{2}\right)$ cancel because of the constraints in Eqs. (9, 13).

The delta resonance plays a special role in the nucleon structure physics. In a world where the number of quark colors $\left(N_{c}\right)$ go to infinity, the delta resonance is degenerate with the nucleon, i.e. $\Delta=M_{\Delta}-M_{N}=\mathcal{O}\left(1 / N_{c}\right)$. If, at the same time, the standard spontaneous chiral symmetry breaking occurs, the chiral corrections to the nucleon properties are strongly affected by the degenerate delta contribution. In the real world where $\Delta \sim 300 \mathrm{MeV}$, the significance of the delta contribution depends strongly on the spin-isospin channel to which an observable belongs [13]. It can be calculated in an effective field theory approach in which $\Delta$ is counted as the same order as $m_{\pi}$ [10,14.

In Fig. 2, we have shown three different possible ways that the delta resonance can contribute as an intermediate state to the spin structure of the nucleon: First, the delta is present when the energy-momentum tensor of the pion contributes (Fig. 2b); second, the delta contributes to the wave function renormalization of the nucleon (Fig. 2c); and finally the energy-momentum tensor of the delta contributes directly (Fig. 2a)

$$
T_{\Delta_{q, g}}^{(\mu \nu)}\left(\mu^{2}\right)=-3 b_{(q, g) \Delta}\left(\mu^{2}\right) i \partial^{\alpha}\left\{\bar{\Delta}^{\beta} v^{(\mu}\left[S^{\nu)}, S_{\alpha}\right] \Delta_{\beta}\right\}+\ldots
$$

Here the leading term contributes to the delta spin in the chiral limit. Indeed, the total spin of the delta leads to the following constraint,

$$
b_{q \Delta}\left(\mu^{2}\right)+b_{g \Delta}\left(\mu^{2}\right)=1 .
$$


The calculation of the delta contribution to $J_{q, g}\left(\mu^{2}\right)$ is straightforward. The combined leading non-analytic chiral correction is

$$
\begin{aligned}
& J_{q, g}\left(\mu^{2}\right)=\frac{1}{2}\left\{b_{(q, g) N}\left(\mu^{2}\right)+3\left[a_{(q, g) \pi}\left(\mu^{2}\right)-b_{(q, g) N}\left(\mu^{2}\right)\right] \frac{g_{A}^{2} m_{\pi}^{2}}{\left(4 \pi f_{\pi}\right)^{2}} \ln \left(\frac{m_{\pi}^{2}}{\Lambda_{\chi}^{2}}\right)\right. \\
& \left.-\left(\frac{9}{2} b_{(q, g) N}\left(\mu^{2}\right)+3 a_{(q, g) \pi}\left(\mu^{2}\right)-\frac{15}{2} b_{(q, g) \Delta}\left(\mu^{2}\right)\right) \frac{\left(2 \sqrt{2} g_{\pi N \Delta}\right)^{2}}{\left(3 \cdot 4 \pi f_{\pi}\right)^{2}} K\left(m_{\pi}, \Delta\right)\right\}+\cdots,
\end{aligned}
$$

where

$$
K\left(m_{\pi}, \Delta\right)=\left(m_{\pi}^{2}-2 \Delta^{2}\right) \log \left(\frac{m_{\pi}^{2}}{\Lambda_{\chi}^{2}}\right)+2 \Delta \sqrt{\Delta^{2}-m_{\pi}^{2}} \log \left(\frac{\Delta-\sqrt{\Delta^{2}-m_{\pi}^{2}+i \epsilon}}{\Delta+\sqrt{\Delta^{2}-m_{\pi}^{2}+i \epsilon}}\right) .
$$

Again, all the delta contributions cancel when summed over quark and gluon operators because of the constraints on the low-energy constants.

Another nontrivial check of the above result is to let the number of colors $N_{c}$ go to infinity. In this limit, the individual nucleon and delta contributions scale as $N_{c}$. However, because $2 \sqrt{2} g_{\pi N \Delta} / 3=g_{A}$ and $b_{(q, g) N}=b_{(q, g) \Delta}$ at large $N_{c}$ [15], the order $N_{c}$ chiral contributions cancel. In the real world, we can use the large- $N_{c}$ approximation of the delta observables to get an estimate of the total chiral contributions,

$$
J_{q, g}\left(\mu^{2}\right)=J_{q}^{0}+\frac{3 g_{A}^{2}}{\left(4 \pi f_{\pi}\right)^{2}}\left(J_{q}^{0}-\frac{\langle x\rangle_{q / \pi}}{2}\right)\left[K\left(m_{\pi}, \Delta\right)-m_{\pi}^{2} \ln \frac{m_{\pi}^{2}}{\Lambda_{\chi}^{2}}\right],
$$

which depends on the unknown $J_{q}^{0}$. It is interesting to note that the non-analytic corrections will be largely cancelled if $J_{q}^{0} \approx\langle x\rangle_{q / \pi} / 2$, a possibility favored by empirical considerations. In this case, a linear $m_{\pi}^{2}$ extrapolation of lattice calculations to the chiral limit might be justified.

In Refs. [16], the quark and gluon angular momentum distributions are found to be $J_{q}(x)=x\left(\Sigma(x)+E_{q}(x, 0,0)\right) / 2$ and $J_{g}(x)=x\left(g(x)+E_{g}(x, 0,0)\right) / 2$, where $\Sigma(x)$ and $g(x)$ are unpolarized (singlet) quark and gluon distributions and $E_{q, g}(x, 0,0)$ are generalized parton distributions [17]. The moments of these distributions are related to the form factors of the twist-two operators [8]. In leading order in $q$,

$$
\begin{aligned}
\left\langle p^{\prime}\left|\mathcal{O}^{\mu_{1} \mu_{2} \ldots \mu_{n}}\right| p\right\rangle= & A_{n, 0}\left(0, \mu^{2}\right) \bar{N}(v) v^{\mu_{1}} v^{\mu_{2}} \ldots v^{\mu_{n}} N(v) M^{n-1} \\
& +\left[A_{n, 0}\left(0, \mu^{2}\right)+B_{n, 0}\left(0, \mu^{2}\right)\right] \bar{N}(v)\left[S^{\mu_{1}}, S \cdot q\right] v^{\mu_{2}} \ldots v^{\mu_{n}} N(v) M^{n-2}+\ldots
\end{aligned}
$$

Then the relation is

$$
\int_{-1}^{1} x^{n-2} J_{q, g}\left(x, \mu^{2}\right) d x=\frac{1}{2}\left[A_{n, 0}\left(0, \mu^{2}\right)+B_{n, 0}\left(0, \mu^{2}\right)\right]
$$

for $n=2,4, \ldots$ By going through the same calculation as above, one can show that,

$$
\begin{aligned}
\int_{-1}^{1} x^{n-2} & J_{q, g}\left(x, \mu^{2}\right) d x=b_{n(q, g) N}\left(\mu^{2}\right)\left(1-3 \frac{g_{A}^{2} m_{\pi}^{2}}{\left(4 \pi f_{\pi}\right)^{2}} \ln \left(\frac{m_{\pi}^{2}}{\Lambda_{\chi}^{2}}\right)\right) \\
-\left(\frac{9}{2} b_{n(q, g) N}\left(\mu^{2}\right)-\frac{15}{2} b_{(q, g) \Delta}\left(\mu^{2}\right)\right) & \frac{\left(2 \sqrt{2} g_{\pi N \Delta}\right)^{2}}{\left(3 \cdot 4 \pi f_{\pi}\right)^{2}} K\left(m_{\pi}, \Delta\right)+\cdots,
\end{aligned}
$$


for $n=4,6, \ldots$, where $b_{n(q, g) N}$ and $b_{n(q, g) \Delta}$ are the coefficients of the nucleon and delta spindependent twist two operators in the effective theory. Apart from the absence of the pion contribution, the other terms are similar to the $n=2$ moment.

To summarize, we have calculated the leading chiral contribution to the spin structure of the nucleon. These results provide interesting insight about the role of the pion in the composition of the nucleon spin. It also provides useful guidance for extrapolating lattice QCD calculations at large quark masses to the chiral limit [7,18].

This work is supported in part by the U.S. Dept. of Energy under grant No. DE-FG0293ER-40762-245. 


\section{REFERENCES}

[1] J. Ashman et al., Phys. Lett. B 202 (1988) 603; Nucl. Phys. B 328 (1989) 1.

[2] F. E. Close, An Introduction to Quarks and Partons, Academic Press, london (1979).

[3] For a review, see B. W. Filippone and X. Ji, hep-ph/0101224, Adv. in Nucl. Phys. Vol. 26, 1 (2001).

[4] X. Ji, Phys. Rev. Lett. 78, 610 (1997); Phys. Rev. D 55, 7114 (1997).

[5] X. Ji, Phys. Rev. D 58, 056003 (1998).

[6] A. V. Radyushkin, Phys. Lett. B 385, 333 (1996); J. C. Collins, L. Frankfurt, and M. Strikman, Phys. Rev. D 56, 2982 (1997).

[7] N. Mathur, S. J. Dong, K. F. Liu, L. Mankiewicz, and N. C. Mukhopadhyay, Phys. Rev. D 62, 114504 (2000); V. Gadyiak, X. Ji, and C. Chung, to be published; X. Ji and I. Balitsky, Phys. Rev. Lett. 79, 1225 (1997).

[8] X. Ji and R. Lebed, Phys. Rev. D 63, 076005 (2001).

[9] S. J. Brodsky, D. Hwang, B.-Q. Ma, I. Schmidt, Nucl. Phys. B 593, 311 (2001).

[10] E. Jenkins and A. V. Manohar, Phys. Lett. B 255, 558 (1991).

[11] J. W. Chen and X. Ji, hep-ph/0105197; D. Arndt and M. J. Savage, nucl-th/0105045; See also, J. W. Chen and X. Ji, Phys. Rev. Lett. 87, 152002 (2001).

[12] M. Gluck, E. Reya and A. Vogt Z. Phys. C53, 651 (1992); M. Gluck, E. Reya and I. Schienbein, Eur. Phys. J. C10, 313 (1999).

[13] T. Cohen, Rev. Mod. Phys. 68, 599 (1996).

[14] T.R. Hemmert, B.R. Holstein and J. Kambor J. Phys. G 24, 1831 (1998).

[15] J. W. Chen and X. Ji, hep-ph/0105296.

[16] P. Hoodbhoy, X. Ji and W. Lu, Phys. Rev. D 59, 014013 (1999); Phys. Rev. D 59, 074010 (1999).

[17] See for example, X. Ji, J. Phys. G 24, 1181 (1998); A. V. Radyushkin, hep-ph/0101225; K. Goeke, M. V. Polyakov, M. Vanderhaeghen, hep-ph/0106012.

[18] W. Detmold, W. Melnitchouk, J. W. Negele, D. B. Renner, and A. W. Thomas, heplat/0103006; A. W. Thomas, W. Melnitchouk, F. M. Steffens, Phys. Rev. Lett. 85, 2892 (2000). 